\title{
Impact of the Number and Quality of Embryos Transferred in Multiple and Single Pregnancies
}

\author{
Thais F. Machado ${ }^{1}$, Patrícia Ramos Guzatti ${ }^{1}$, Daniela Bragaa, ${ }^{2,}$, Edson Borges Jr ${ }^{2,3}$, Raquel Gomes Aguiar da Silva ${ }^{1}$ \\ ${ }^{1}$ University of Southern Santa Catarina - UNISUL, SC, Brazil \\ ${ }^{2}$ Fertility Medical Group, São Paulo, Brazil \\ ${ }^{3}$ Sapientiae Institute - Centro de Estudos e Pesquisa em Reprodução Assistida, São Paulo, Brazil
}

\begin{abstract}
Objective: To investigate the relation between maternal age and embryo quality in choosing how many embryos should be transferred to obtain high pregnancy rates with minimal risk of multiple gestations.

Methods: Cross-sectional study, with secondary data analysis, including infertile couples undergoing Intracytoplasmic Sperm Injection, between 2005-2013, in the Assisted Fertilization Center - Fertility. The data was analyzed using the SPSS 16.0, in a descriptive and bivariate way using the chi-square test, $P<0.05$. Approved by the CEP UNISUL.

Results: $54.8 \%$ of pregnancies occurred with women $\leq 35$ years. The prevalence of single pregnancy was 2.42 times the multiple pregnancies and no significant increase in multiplicity in the transfer of 3 or 4 embryos. The percentage of positive pregnancies was prevalent in the age group $\leq$ 35 years, regardless of embryo quality. Regardless of age, the greater the number of high quality transferred embryos, the higher the multiplicity. This was not observed with low-quality embryos. With high quality embryos, the prevalence of positive pregnancies in middle age women was $9 \%$ lower when compared to those aged $\leq 35$ years, with a $P=0,310$. With low-quality embryos, the prevalence of positive pregnancy in this age group was $39 \%$ lower when compared to those aged $\leq 35$ years, with a $P<0.01$.

Conclusion: The greater the number of high quality transferred embryos, the higher the multiplicity. However, this relationship is not true for low quality embryos. When only high quality embryos are transferred, the patients with intermediate age have similar pregnancy results to those from younger patients. Already with low-quality embryos, this same age group presents similar pregnancy results to older patients.
\end{abstract}

Keywords: Assisted reproduction, Embryo quality, Embryo transfer, Multiple pregnancies

\section{INTRODUCTION}

A multiple gestation is the most common iatrogenic complication of Assisted Reproductive Techniques (ART), being responsible for the increase in the incidence of maternal, fetal, perinatal, newborn and child morbidity and mortality (Lee et al., 2006; Paperstraten et al., 2008; Gerris, 2009; Graner \& Barros, 2009; Luke et al., 2010). Among the complications related to the newborn, the rates of stillbirth, low birth weight and neurological disabilities are the most frequent (Kalu et al., 2008; Gelbaya et al., 2010; Barri et al., 2011; Sullivan et al., 2012). Among the most frequent maternal complications we have hypertension, gestational diabetes and post partum hemorrhage (Kalu et al., 2008). Moreover, because of the greater likelihood of complications during pregnancy, we have higher rates of prematurity and preterm labor (PPT) (Practice Committee of American Society for Reproductive Medicine, 2012).

In addition to the clinical problems, multiple gesta- tions also cause major economic and social consequences (Lee et al., 2006; Kalu et al., 2008; Paperstraten et al., 2008; Gerris, 2009; Kutlu et al., 2011; Martini et al., 2011; Lawlor \& Nelson, 2012; Practice Committee of American Society for Reproductive Medicine, 2012). High costs are related to maternal hospitalization, cesarean section and neonatal intensive care, the most frequent (Kalu et al., 2008; Practice Committee of American Society for Reproductive Medicine, 2012). The long-term costs are associated with chronic diseases, rehabilitation and investment in special education (Practice Committee of American Society for Reproductive Medicine, 2012).

For these reasons, experts have tried to reduce the number of embryos transferred per cycle in order to reduce multiplicity without affecting pregnancy rate (Kalu et al., 2008; Kutlu et al., 2011; Practice Committee of American Society for Reproductive Medicine, 2012). In some places, guidelines have been created to suggest the number of embryos to be transferred according to patient age, embryo quality and failure in previous proceedings (Kalu et al., 2008; Bromer et al., 2009; Kutlu et al., 2011; The Practice Committee of the American Society of Reproductive Medicine and the Practice Committee of the Society for Assisted Reproductive Technology, 2013). Elsewhere, laws were created forcing assisted reproduction clinics to reduce this number, often times being mandatory to transfer a single embryo (Lee et al., 2006; Kalu et al., 2008; Kutlu et al., 2011; The Practice Committee of the American Society of Reproductive Medicine and the Practice Committee of the Society for Assisted Reproductive Technology, 2013). In Brazil, there are no laws, and just a handful of guidelines. There is a resolution of the Federal Council of Medicine (Conselho Federal de Medicina - CFM - no 2013/13) which limits the number of embryos to be transferred depending, solely, on the age of the woman (CFM, 2013).

Thus, this work aims to investigate the relation between maternal age and embryo quality in choosing how many embryos should be transferred to obtain high pregnancy rates with minimal risk of multiple gestations.

\section{MATERIALS AND METHODS}

This is a retrospective cross-sectional study. We analyzed information from all infertile couples undergoing Intracytoplasmatic Sperm Injection (ICSI) between January $1^{\text {st }} / 2005$ and December 31st/2013; totaling 2649 couples, in a private center of Assisted Human Reproduction.

We included in the study all infertile couples, characterized by their inability of pregnancy after one year of regular sexual intercourse without using contraception and undergoing ICSI.

Within 10 days after the embryo transfer, we carried out a quantitative dosage of serum levels of the $B$ subunit of human chorionic gonadotropin (B-hCG), indicative of positive pregnancy. Clinical pregnancy was confirmed through the detection of the gestational sac and the fetal heartbeat in a transvaginal pelvic ultrasound performed by 3 to 4 weeks after the embryo transfer. The 
number of gestational sacs defined the implantation rate with heartbeat divided by the total number of embryos transferred.

We took off the infertile couples who had, at the end of the procedure, multiple monozygotic pregnancies, pregnancy from egg donation and/or frozen material, absence of embryo transfer, ectopic or biochemistry pregnancy, abortion without identification of gestational sac or that did not show the variables of interest to the study in the database, namely, women's age at the puncture of oocytes, embryo quality, number of gestational sacs on transvaginal pelvic ultrasonography and number of embryos transferred.

The embryo quality classification was based on criteria determined in an Expert Meeting on Assisted Reproduction, composed of members of the European Society of Human Reproduction and Embryology (ESHRE), held in Istanbul, which defined a globally accepted consensus (Alpha scientists in Reproductive Medicine and ESHRE Special Interest Group of Embryology, 2011). Basically, classify embryonic evaluation by days of evolution. Thus, on Day 0 , to evaluate characteristics of the oocytes such as molecular anatomy and cell cytoplasm. On Day 1, fertilization and zygote. Fertilization is assessed by the presence of two pro nuclei and the formation of the second polar body; the zygote is analyzed for the presence of early and pronuclear morphology cleavage. On days 2, and 3, evaluating fragmentation, multinucleation, cleavage rate and cell size. On Day 4, morula stage, we observed the degree of compression between cells. Now, on Day 5 blastocyst stage, the importance is in the inner cell mass and firmly adhered to the presence of hatching, hatching blastocyst in the culture medium. Each embryo is evaluated in each of these criteria, the higher the score, the better the quality.

The medical records of the patients included in this study were filed, in addition to being available for consultation in digitized format. All clinical and laboratory data relating to each of the procedures performed were entered in an electronic database (Access format) by qualified and properly trained personnel.

The electronic database was converted to an Excel spreadsheet for statistical analysis purposes in SPSS 16.0. Descriptive analysis was performed initially and after, a bivariate analysis was carried out between the independent variables and the outcome variable for single or multiple gestations. For the bivariate analysis, we used the Chisquare test for comparison of two or more proportions with a significance level of $5 \%$ (value of $P<0.05$ ). The measure of Association used was the prevalence ratio (PR) with $95 \%$ confidence interval.

This work is free from conflict of interest between researchers and participants. Data collection was started after approval of the Committee of Ethics in Research (CER) with Human Beings of the University of Southern Santa Catarina (UNISUL), as opinion embodied number 527.059.

\section{RESULTS}

\section{General Data}

There were 2,649 couples participating in this study. Of these, 417 were excluded because they did not submit their embryonic transfer; 28, for having an abortion without identification of the gestational sac; 12 , by ectopic gestation and 25, for biochemical pregnancy, totaling 482 excluded couples, which resulted in the inclusion of 2,167 couples. Of these, 1,421 didn't get pregnant, while 746 had their pregnancies confirmed by positive B hCG associated with the identification of the gestational sac.

According to age distribution of women undergoing ICSI, $46.8 \%$ were aged $\leq 35$ years at the moment of oo- cyte puncture. While the percentages of intermediate age groups of $\geq 40$ years were similar $(27.6 \%$ vs. $25.6 \%)$.

Among couples with positive pregnancy, $54.8 \%$ were aged $\leq 35$ years at the time of oocyte puncture (table 1 ).

\begin{tabular}{|l|c|c|}
\hline \multicolumn{3}{|l|}{$\begin{array}{l}\text { Table 1. Distribution of the women's age accord- } \\
\text { ing to the gestational outcome }\end{array}$} \\
\hline & $\begin{array}{c}\text { Negative pregnancy } \\
\mathrm{n}(\%)\end{array}$ & $\begin{array}{c}\text { Positive pregnancy } \\
\mathrm{n}(\%)\end{array}$ \\
\hline Age (years) & & $409(54.8)$ \\
\hline$\leq 35$ & $605(42.6)$ & $197(26.4)$ \\
\hline 36 a 39 & $402(28.3)$ & $140(18.8)$ \\
\hline$\geq 40$ & $414(29.1)$ &
\end{tabular}

Prevalence of single and multiple gestations according to age and number of embryos transferred

The prevalence of single gestations, when two embryos were transferred was 2.42 times that of multiple gestations. When three embryos were transferred, it was 1.88 times that of a single gestation. And with the transfer of four embryos, this prevalence was 2 times higher $(P<0.01)$. However, there was no statistical difference in the rate of single gestation when we compared the transfer of three and four embryos with two embryos (Table 2).

\begin{tabular}{|c|c|c|c|c|}
\hline & $\begin{array}{c}\text { Single } \\
\text { gestation } \\
\text { n (\%) }\end{array}$ & $\begin{array}{c}\text { Multiple } \\
\text { gestation } \\
\text { n (\%) }\end{array}$ & PR & Total \\
\hline \multicolumn{5}{|c|}{ No embryos } \\
\hline 1 & $53(100)$ & - & - & 53 \\
\hline 2 & $288(70.8)$ & $119(29.2)$ & $2.42 *$ & 407 \\
\hline 3 & $167(65.2)$ & $89(34.8)$ & $1.88 *$ & 256 \\
\hline 4 & $20(66.7)$ & $10(33.3)$ & $2.00 *$ & 30 \\
\hline Total & 528 & 218 & - & 746 \\
\hline
\end{tabular}

$* P<0.01$

Prevalence of single and multiple gestations in accordance with the age and quality of embryos transferred

When were transferred high quality embryos, there was also no significant difference in gestation outcome of when patients aged between 36 and 39 years when compared to those $\leq 35$ years (PR 0.91). However, when compared with patients $\geq 40$ and $\leq 35$ years, the prevalence of positive pregnancy was $28 \%$ lower in older patients (PR $0.72)(P<0.01)$. Within the comparison of age groups of 36 to 39 and $\geq 40$ years, we obtained $21 \%$ (PR 0.79 ) of positive pregnancies, lower in older patients, with a $p$ value of $<0.05$ (Table 3 ).

When poor-quality embryos were transferred, the prevalence of positive pregnancy within the age range of 36 and 39 years was 39\% lower when compared to that of the age group $\leq 35$ years (PR 0.61), $P<0.01$.

This same significance was observed when we compared the age groups $\geq 40$ and $\leq 35$ years, in which the prevalence of positive pregnancies was $38 \%$ lower in older patients (PR 0.62 ). However, with the comparison between the ages of 36 to 39 and $\geq 40$ years, there was no prevalence of positive pregnancies between ages (PR 1.02), $P=0,925$ (Table 3). 
Table 3. Comparison of pregnancy outcomes among age groups according to embryo quality

\begin{tabular}{|c|c|c|c|c|c|c|}
\hline & & $\begin{array}{l}\text { Positive } \\
\text { pregnancy } n(\%)\end{array}$ & PR & $\begin{array}{l}\text { Negative } \\
\text { pregnancy } \mathrm{n}(\%)\end{array}$ & PR & Total \\
\hline \multirow[t]{2}{*}{ Quality } & Age group (years) & & & & & \\
\hline & $\leq 35$ & $284(47)^{*}$ & 1.00 & $323(53)^{*}$ & 1.00 & 607 \\
\hline \multirow[t]{3}{*}{ High } & 36 a 39 & $123(43) \dagger$ & 0.91 & $162(57) \dagger$ & 1.07 & 285 \\
\hline & $\geq 40$ & $75(34)+$ & 0.72 & $142(66)+$ & 1.24 & 217 \\
\hline & $\leq 35$ & $125(31.0)^{*}$ & 1.00 & $282(69.0) *$ & 1.00 & 407 \\
\hline \multirow[t]{2}{*}{ Low } & 36 a 39 & $74(19.0)^{*}$ & 0.61 & $314(81.0) *$ & 1.17 & 388 \\
\hline & $\geq 40$ & $65(19.4)^{*}$ & 0.62 & $271(80.6)^{*}$ & 1.16 & 336 \\
\hline
\end{tabular}

$* P<0.01+P<0.05$

Prevalence of single and multiple gestations with high quality embryo transfer according to age

With the transfer of two high quality embryos in the group-aged $\leq 35$ years, we had $30.7 \%$ of multiple gestations. Now, when three high-quality embryos were transferred, there was an increase of $32 \%$ in the multiple gestation rate, with a $P=0.009$. With the transfer of four embryos, we had $100 \%$ of single gestations, representing only 2 cases, and therefore not included in the test of linearity (Table 4).

With the transfer of two of high quality embryos in the group aged between 36 and 39 years, we had 31.3 of multiple gestations. When three high-quality embryos were transferred, there was a $17 \%$ increase in multiplicity. With four embryos transferred, this same rate was 2.66 times higher when compared with the transfer of two embryos. Comparing three and four embryos, we found a prevalence of 2.27 times more multiple gestations ( $83.3 \%$ vs. 36.6$)$, as more embryos were transferred. $(P=0.004)$ (Table 4$)$.

With the transfer of two high quality embryos in the group aged $\geq 40$ years, we had $4 \%$ of multiple gesta- tions. When three high-quality embryos were transferred, there was an increase of 3.8 times in multiplicity. With four embryos, the prevalence of multiple gestations was 8.32 times that of two embryos. Comparing three and four embryos, we found a prevalence of 2.2 times more multiple gestations (33.3\% vs. $15.2 \%)$ when more embryos were transferred. $(P=0.001)$ (Table 4$)$.

Prevalence of single and multiple pregnancies with low-quality embryo transfer according to age

With the transfer of two low quality embryos in the group aged $\leq 35$ years, we had $30 \%$ of multiple gestations. When three low-quality embryos were transferred, there was an increase of $21 \%$ in multiplicity, $(P=0.470)$. When four embryos were transferred, we had $100 \%$ of single gestations, representing only 2 cases, and therefore not included in the linearity test (Table 5).

With the transfer of two low quality embryos in the group aged between 36 and 39 years we had $34.5 \%$ of multiple gestations. When three low-quality embryos were transferred, in this same group, there was a $26 \%$ drop

\begin{tabular}{|c|c|c|c|c|c|c|}
\hline & & $\begin{array}{c}\text { Single gest } \\
\mathrm{n}(\%)\end{array}$ & PR & $\begin{array}{c}\text { Multiple gestation } \\
\text { n (\%) }\end{array}$ & PR & P* Value \\
\hline \multirow[t]{2}{*}{ Age group } & No Embryos & & & & & \\
\hline & 1 & $13(100)$ & - & $0(00.0)$ & - & 0.009 \\
\hline \multirow[t]{4}{*}{$\leq 35$ years } & 2 & $142(69.3)$ & 1.00 & $63(30.7)$ & 1.00 & - \\
\hline & 3 & $38(59.4)$ & 0.85 & $26(40.6)$ & 1.32 & - \\
\hline & 4 & $2(100)$ & - & $00(00.0)$ & - & - \\
\hline & 1 & $9(100)$ & - & $0(00.0)$ & - & 0.004 \\
\hline \multirow[t]{4}{*}{36 e 39 years } & 2 & $46(68.7)$ & 1.00 & $21(31.3)$ & 1.00 & - \\
\hline & 3 & $26(63.4)$ & 0.92 & $15(36.6)$ & 1.17 & - \\
\hline & 4 & $1(16.7)$ & 0.24 & $5(83.3)$ & 2.66 & - \\
\hline & 1 & $11(100)$ & - & $0(00.0)$ & - & 0.001 \\
\hline \multirow[t]{3}{*}{$\geq 40$ years } & 2 & $24(96.0)$ & 1.00 & $1(04.0)$ & 1.00 & - \\
\hline & 3 & $28(84.8)$ & 0.88 & $5(15.2)$ & 3.80 & - \\
\hline & 4 & $4(66.7)$ & 0.69 & $2(33.3)$ & 8.32 & - \\
\hline
\end{tabular}

* $P$ for linear trend test (chi-square Pearson) 


\begin{tabular}{|c|c|c|c|c|c|c|}
\hline & & Single ge & tion & Multiple ge & tion & \\
\hline & & n (\%) & PR & n (\%) & PR & $P *$ Value \\
\hline Age group & No Embryos & & & & & \\
\hline & 1 & $8(100)$ & - & $0(00.0)$ & - & - \\
\hline$\leq 35$ years & 2 & $42(70.0)$ & 1.00 & $18(30.0)$ & 1.00 & 0.470 \\
\hline & 3 & $35(63.6)$ & 0.90 & $20(36.4)$ & 1.21 & - \\
\hline & 4 & $2(100)$ & - & $0(00.0)$ & - & - \\
\hline & 1 & $5(100)$ & - & $0(00.0)$ & - & - \\
\hline 36 e 39 years & 2 & $19(65.5)$ & 1.00 & $10(34.5)$ & 1.00 & 0.432 \\
\hline & 3 & $29(74.4)$ & 1.13 & $10(25.6)$ & 0.74 & - \\
\hline & 4 & $1(100)$ & - & $0(00.0)$ & - & - \\
\hline & 1 & $7(100)$ & - & $0(00.0)$ & - & - \\
\hline$\geq 40$ years & 2 & $15(71.4)$ & 1.00 & $6(28.6)$ & 1.00 & 0.893 \\
\hline
\end{tabular}

* $P$ for linear trend test (chi-square Pearson)

in multiplicity, with $P=0.432$. When four embryos were transferred, we had $100 \%$ of single gestations, representing only 1 case, and therefore not included in the linearity test (Table 5).

With the transfer of two low quality embryos in the group aged $\geq 40$ years, we had $28.6 \%$ of multiple gestations. When three and four low-quality embryos were transferred, there was a fall of $27 \%$ and $46 \%$ in multiplicity, respectively, when compared to the rates of transfer of two embryos. Comparing three and four embryos, we had a fall rate of $26 \%$ ( $15.4 \%$ vs. $20.8 \%$ ) in multiplicity when more embryos were transferred. It is important to notice that all values mentioned above are not statistically significant $(P=0.89)$ (Table 5).

\section{DISCUSSION}

Based on data from approximately 10 years performing ICSI, this study showed that the vast majority of patients who seek treatment to get pregnant, were aged $\leq 35$ years at the time of oocytes puncture. While the percentages of those aged $\geq 40$ years were similar. These same proportions were found in a study by Graner \& Barros (2009), evidencing $45.8 \%$ of patients aged $\leq 35$ years, $29 \%$ between 36 and 39 and $25.2 \%, \geq 40$ years. According to Braga et al. (2015), in a study with 1,497 couples undergoing ICSI, $46.49 \%$ were aged $\leq 35$ years at the time of puncture. The age groups considered separately, showed a prevalence of younger women. However, what is observed is that more than $50 \%$ in all the references cited, including in this study, covers women with age greater than 35 years. This fact is entirely related to modern society, i.e. women investing in career and postponing motherhood.

When associating age with positive pregnancy rates, we found a majority of pregnancies occurring at age $\leq$ 35 years, followed by intermediate age and, lastly, $\geq 40$ years. These results were very similar to those obtained by RedLara, with the exception of the intermediate band. The rates observed in recent years, for this institution, were: $42 \%$ for patients $\leq 34$ years, $35 \%$ for those between 35 and 39 years and $20 \%$ for patients $\geq 40$. In relation to the range 36 to 39 , the rate found in the current study was somewhat lower than that of RedLara ( $26.4 \%$ vs. $35.0 \%)$ because the range of this one includes patients with 35 years (Zegers-Hochschild et al., 2013). This data shows that the woman's age is an important prognostic factor for positive pregnancy outcomes. And, even with the biological difficulty in this context, with the advancement of medicine, it is possible to reach $45 \%$ of pregnancies in older patients.

The relationship between the number of embryos transferred and single and multiple gestations denoted a significant increase of $29.2 \%$ in multitude with the transfer of 2 embryos. However, the significance was not viewed with the transfer of 3 or 4 embryos. These findings were also observed by RedLara (Zegers-Hochschild et al., 2013), which featured multiple gestation rates increase of $22.4 \%$ with the transfer of 2 embryos. With 3 or 4 embryo transfers, there was no significant difference. We, then, concluded that the increase in the number of embryos transferred does not necessarily correlate with the likelihood of pregnancy, being relevant to consider embryo quality. When only low quality embryos are available, there is a tendency to transfer more, however the potential for implantation of each embryo is directly related to its quality.

Thus, according to the relationship between embryo quality and pregnancy, high quality embryos transferred were much more frequent in positive pregnancies when compared to the negative ones. Already low-quality embryos were mostly present in the negative pregnancies. This data shows the importance of embryo quality as a prognostic factor, since it is the morphology of the embryo that is responsible for predicting their ability to deploy (Tiitinen et al., 2012; Braga et al., 2015).

When they compared the results of pregnancies between age groups with high and low quality embryo transfers, they approached two important questions that are presented below.

The percentage of positive gestation at the age group $\leq 35$ years was prevalent, regardless of embryo quality. This data was confirmed by Braga et al. (2015) that showed $35.9 \%$ of positive pregnancies with high quality embryos and $35.6 \%$ of low quality embryos in patients aged $\leq 36$ years. When the other two age groups were analyzed, they found a very important drop in the rate of pregnancies with low quality embryo transfer. However, this data was different from that of the comparative study 
cited above. According to Braga et al. (2015), there was no significant difference in the rate of pregnancies with low or high quality embryo transfer for patients aged $\geq 36$ years (31\% vs. $31.9 \%)$. However, Lee et al. (2006), in a retrospective study carried out with 584 ICSI cycles, from 2001 to 2004, at the Taiwan University Hospital, showed $49.5 \%$ and $25.8 \%$ of positive pregnancies with high quality embryos in young and older subjects, respectively. This data suggests that embryo quality is more important than age in order to obtain positive gestation among young patients, i.e. $\leq 35$ years. On the other hand, age becomes a more important prognostic factor than embryo quality for patients aged $>35$ years.

In addition, in this study we found that with the transfer of high quality embryos, positive pregnancy rates in intermediate ages, was very close to that of the lower age group. Which did not occur with patients over 40 years, which showed a drop in the rate of positive pregnancies. With low-quality embryo transfers, the rate of pregnancy presents an important decline with increasing age, showing values very close between 36 to 39 and $\geq 40$ years. This data shows that, depending on embryo quality; the intermediate age range shall have gestation rates similar to that of younger or older patients. I.e., when only high-quality embryos are transferred, the patients aged between 36-39 years had approximately the same pregnancy rate that patients aged $\leq 35$ years. With poor-quality embryos, this same age group had a gestation rate similar to that of older patients.

When we joined the number of high-quality embryos transferred in each age group, with single and multiple gestations we found that, regardless of age, the greater the number of embryos transferred, the greater the rate of multiple pregnancies. According to Bhattacharya \& Kamath (2014), in a revision study, presented data from Cochrane, reviewed by Pandian et al., and data from the retrospective study of Licciardi et al., characterizing that multiple pregnancies are more frequent with the transfer of 3 than with 2 embryos of high quality (30\% vs. $0 \%$ and $51 \%$ vs. $40.5 \%$, respectively). This is because the implantation capacity of an embryo is directly related to its morphology. That is, the better the quality, the greater its ability to implant.

When we mixed the number of low-quality embryos transferred in each age group, with single and multiple gestations, it was noted that, with the transfer of $3 \mathrm{em}-$ bryos in patients aged $\leq 35$ years, there was a non-significant increase in the rate of multiple gestations. Already in the intermediate range, we found a drop in the rate of multiple pregnancies with the transfer of 3 embryos. The same occurred with patients aged $\geq 40$ years. According to Gerris (2009), in a mathematical model developed to check the likelihood of multiple gestations, considering the rate of implantation in $30 \%$, there are multiple gestation rates of $10 \%, 22 \%$ and $40 \%$ with the transfer of 2,3 and 4 embryos, respectively. Murray \& Norman (2014) found $37.7 \%$ and $>45 \%$ of multiple pregnancies with the transfer of 2 and more than 3 embryos, respectively. However, the studies cited above are extremely comprehensive, not featuring embryo quality nor age of the woman in puncture time, such data is extremely relevant for choosing how many embryos to transfer, since they are directly related to its ability to implant. In the study under discussion, even increasing the number of embryos transferred, when low quality, has not increased multiple pregnancy rates. This happened, most likely, because embryo implantation capacity is inversely proportional to the woman's age at the time of oocyte puncture.

Thus, it was possible to identify the relationship between age, number and quality of embryos with simple and multiple gestation rates. These findings are important to prioritize prognostic factors in choosing how many embryos should be transferred in order to obtain high pregnancy rates with minimal risk of multiple gestations.

\section{CONCLUSIONS}

When not considering embryo quality, there is no significant increase in multiplicity with the transfer of more embryos. Considering embryo quality, the greater the number of high-quality embryos transferred, the greater the rate of multiple pregnancies. However, this relationship is not true for low-quality embryos. In addition, when only high-quality embryos are transferred, the patients at intermediate age have gestational results similar to those of younger patients. Already with poor-quality embryos, this same age group presents gestational result similar to those from older patients.

\section{CONFLICT OF INTERESTS}

No conflict of interest have been declared.

\section{Corresponding author:}

Thais F. Machado

University of Southern Santa Catarina

UNISUL, Av. Pedra Branca

Palhoça/SC - Brazil

E-mail: tfmachado@hotmail.com

\section{REFERENCES}

Alpha scientists in Reproductive Medicine and ESHRE Special Interest Group of Embryology. The Istanbul consensus workshop on embryo assessment: proceedings of an expert meeting. Hum Reprod. 2011; 26: 1270-83.

Barri PN, Coroleu B, Clua E, Tur R. Prevention of prematurity by single embryo transfer. J Perinat Med. 2011; 39:23740.

Bhattacharya S, Kamath MS. Reducting multiple births in assisted reproduction technology. Best Pract Res Clin Obstet Gynaecol. 2014; 28:191-99.

Braga DPAF, Setti AS, Figueria RCS, Iaconelli Jr A, Borges Jr E. The impact of the embryo quality on the risk of multiple pregnancies. Zygote. 2015; 23: 662-8.

Bromer JG, Sakkas D, Siano LJ, Benadiva CA, Patrizio P. Reproductive efficiency of women over the age 40 and the low risk of multiple pregnancies. Reprod Biomed Online. 2009; 19:39-45.

CFM - Conselho Federal de Medicina. Resolução CFM no 2013/2013. [Internet]. 2013 [Acesso em 2013 Mai 15]. Available at: http://www.portalmedico.org.br/resolucoes/ CFM/2013/2013_2013.pdf

Gelbaya TA, Tsoumpou I, Nardo LG. The likelihood of live birth and multiple birth after single versus double embryo transfer at the cleavage stage: a systematic review and meta-analysis. Fertil Steril. 2010; 94:936-45.

Gerris J. Single-embryo transfer versus multiple-embryo transfer. Reprod Biomed Online. 2009; 182:S63-70.

Graner VR, Barros SMO. [Maternal complications and neonatal events associated to multiple pregnancies resulting from assisted reproduction techniques]. Rev Enferm USP. 2009; 43:103-9.

Kalu E, Thum MY, Abdalla H. Reducing multiple pregnancy 
in assisted reproduction technology: towards a policy of single blastocyst transfer in younger women. BJOG. 2008; 115:1143-50.

Kutlu P, Atvar O, Vanlioglu OF, Kutlu U, Arici A, Yilmaz S, Yilmaz E, Delikara N, Bener F, Kamar A, Alpak O, Ozekici $U$. Effect of the new legislation and single-embryo transfer policy in Turkey on assisted reproduction outcomes: preliminary results. Reprod Biomed Online. 2011; 22: 208-14.

Lawlor DA, Nelson SM. Effective of age on decisions about the numbers of embryos to transfer in assisted conception: a prospective study. Lancet. 2012; 379:521-7.

Lee TH, Chen CD, Tsai YY, Chang LJ, Ho HN, Yang YS. Embryo quality is more important for younger women whereas age is more important for older women with regard to in vitro fertilization outcome and multiple pregnancy. Fertil Steril. 2006; 86:64-9.

Luke B, Brown MB, Grainger DA, Cedars M, Klein N, Stern JE. Practice patterns and outcome with the use of single embryo transfer in the United States. Fertil Steril. 2010; 93:490-8.

Martini S, Voorhis BJV, Stegmann BJ, Sparks AET, Shochet $T$, Zimmerman MB, Ryan GL. In vitro fertilization patients support a single blastocyst transfer policy. Fertil Steril. 2011; 96: 993-7.

Murray SR, Norman JE. Multiple pregnancies following assisted reproductive technologies - A happy consequence or double trouble? Semin Fetal Neonatal Med. 2014;19:222-7.

Paperstraten AMV, Nelen WLDM, Hermens RPMG, Jansen $L$, Scheenjes E, Braat DDM, Grol RP, Kremer JA. Why don't we perform elective single embryo transfer? A qualitative study among IVF patients and professionals. Hum Reprod. 2008; 23:2036-42.

Practice Committee of American Society for Reproductive Medicine. Multiple gestation associated with infertility therapy: an American Society for Reproductive Medicine Practice Committee opinion. Fertil Steril. 2012; 97: 825-34.

Sullivan EA, Wang YA, Hayward I, Chambers GM, Illingworth P, McBain J, Norman RJ. Single embryo transfer reduces the risk of perinatal mortality, a population study. Hum Reprod. 2012; 27:3609-15.

Tiitinen A. Prevention of multiple pregnancies in infertility treatment. Best Pract Res Clin Obstet Gynaecol. 2012; 26:829-40.

The Practice Committee of the American Society Reproductive Medicine and the Practice Committee of the Society for Assisted Reproductive Technology. Criteria for number of embryos to transfer: a committee opinion. Fertil Steril. 2013; 99:44-6.

Zegers-Hochschild F, Schwarze JE, Crosby JA, Musri C, Souza MCBS. Assisted reproductive technologies (ART) in Latin America: The Latin American Registry, 2011. JBRA Assist Reprod. 2013; 17:216-23. 